\title{
Drilling azimuth gamma embedded design
}

\author{
Yi Ren ZHOU ${ }^{\mathrm{a}}$, Xiao Lin QIU, Zhi Qiang GUO, Jian Hui ZHANG \\ Nanchang Institute of Technology, No.901 Heroe Ave, Qingshanhu ,Nanchang ,Jiangxi , 330044.China
}

\begin{abstract}
Embedded drilling azimuth gamma design, the use of radioactive measuring principle embedded gamma measurement while drilling a short section analysis. Monte Carlo method, in response to the density of horizontal well logging numerical simulation of 16 orientation, the orientation of horizontal well analysed, calliper, bed boundary location, space, different formation density, formation thickness, and other factors inclined strata dip the impact by simulating 137Cs sources under different formation conditions of the gamma distribution, to determine the orientation of drilling density tool can detect window size and space, draw depth of the logging methods. The data $360^{\circ}$ azimuth imaging, image processing method to obtain graph, display density of the formation, dip and strata thickness and other parameters, the logging methods obtain real-time geo-steering. To establish a theoretical basis for the orientation density logging while drilling method implementation and application of numerical simulation in-depth study of the MWD azimuth and density log response factors of horizontal wells.
\end{abstract}

Keywords: Embedded; LWD; azimuth gamma; numerical simulation; Monte Carlo method

\section{Introduction}

The design of embedded MWD gamma position to msp430 embedded MWD gamma position consider to be the carrier, industrial-oriented research applications can accurately detect the formation and reserve evaluation of the success of intelligent systems, this design makes a great contribution to solve the problem of the introduction of the high cost of foreign advanced LWD system.

From the $20^{\text {th }}$ century of $60 \mathrm{~s}$, it has developed for 80 years, along with the birth of the drilling position with thedensity logging tool and logging technology which had bring a new impetus, they think of the resistivity logging while drilling technology, and nuclear porosity parameters LWD technology, acoustic parameters LWD technology, nuclear magnetic resonance logging-whiledrilling technologies make a progress in drilling industry. However, the development is going through some bottlenecks, with foreign logging-while-drilling density of a Monte Carlo simulation (Monte Carlo) appear that takes hope to break through these bottlenecks . But we still fall behind in comparison with foreign transmission technology, and we still as a freshman at the research work in the azimuth gamma density in this area, Monte Carlo model can be effectively in recording photon radialand longitudinal formations. Monte Carlo model Research would make a progress in the drilling orientation density logging, the instrument has been designed for detecting and playing a important role in the promotion, and to promote the orientation density

\footnotetext{
${ }^{\text {a }}$ Corresponding author: renyiz@126.com

Jiangxi Education Fund projects, Numbering: GJJ151174
}

loggingwhile drilling application and development of the oil well logging industry.

LWD with wired and wireless are two types of transmission. There are cable transmission, special drill pipe transmission and optical transmission ${ }^{[1]}$. The transmission rate is about $2 \mathrm{Mbit} / \mathrm{s}^{[2]}$; wireless transmission isbased on using mud pulse, electromagnetic and acoustic waves ${ }^{[3]}$, etc. ,Such as Baker Hughes who launched overseas drilling focused electrical imaging logging tool ${ }^{[1][3]}$, the logging tool is adapted to different salinity of the mud,and the application get a big success; but there is a big gap in the domestic research in this area."Embedded-based msp430 drilling position Gamma" willdistinguish the reservoirs in different directions, and the interpretation of gamma intensity in different directions of information storage layer boundary judgment, judgment horizon, interpretation, etc. It provide a piercing direction Technical solutions.

\section{Embedded drilling azimuth gamma hardware integration}

Embedded drilling azimuth gamma instrument is consist of the sector detection module, gamma counting module, the gamma sensor module.

The sector detection module by the trysail accelerometer or 3-axis fluxgate and a control circuit. It will produce centrifugal force during rotation, it will cause a bit of impact on the three-axis plus table, so I chose trysail fluxgate sector detection as the sample 
instrument. MSP430 processor control the circuit, it is shown in Figure 3.1, the peripheral circuit includes a serial Flash, ferroelectric memory, 485 communication port, RTC (real-time controller always) circuit. The control circuit, by connecting the SPI with the trysail flux gate, to complete configuration and data read controlcircuit to do the relevant calculation, the current high-side position, gamma counting module through port 485 sent. The main function of the sector detection module is the drilling plane which is separated several sectors, It is divided into four sectors up and down at least, and no more than 16 or 32 sectors.

Gamma counting module uses MSP430 as the processor (as Figure 1), $8 \mathrm{MHz}$ frequency, surrounding with serial Flash $4 \mathrm{M}$, story data; ferroelectric memory, data is temporarily stored; 485 communicated with the sector detection module and the MWD module; it also equippedwith vibration and temperature sensors to detect underground anomalies. Azimuth sensor is packaged by alloy ( usually tungsten alloy), only a window was left in one direction, the current gamma design, all came from the window which is in the direction of where the ground; and while in which direction the current window, you need to provided by the sector detection module.

Gamma Sensor: Use CBG-DGA gamma sensor with $75^{\circ}$ window, gamma range 0-500API, working temperature $0-150{ }^{\circ} \mathrm{C}$.

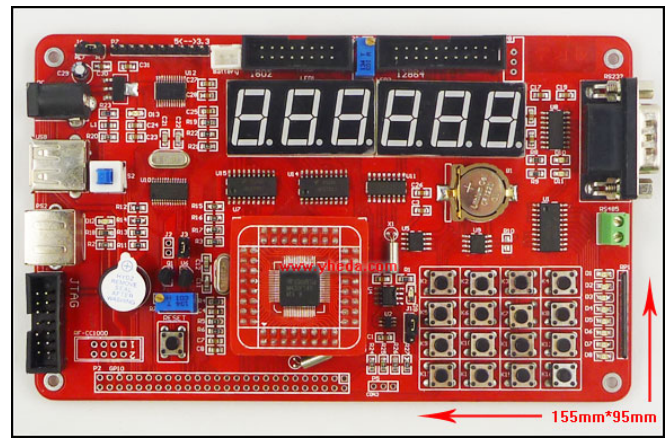

Figure 1 MSP430 processor

\section{LWD azimuth gamma software architecture}

Software hierarchy shown in Figure 2

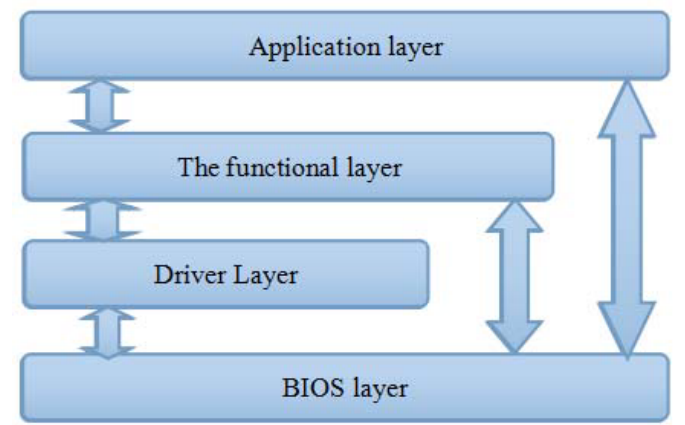

BIOS layer: a package of basic hardware operation, the operation of the register of a transparent, provide a common call interface. In accordance with the functional components it was classified; all features BIOS level debugging is complete, generate a library; the applicationin the formof libraries to link calls.

To use the BIOS module:

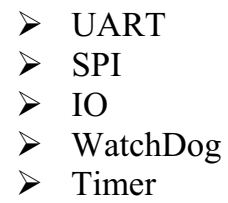

ii. Driver layer: for packaging features provide module-specific features function call entry, driver layer for the control chip is through BIOS calls to achieve, function driver layer may be collected and summarized and logical form comparison of standard call interface, after successful commissioning alone generate library functions, application layer drive functionality in the form of library functions to call. Use Table 1 to the drive module

Table 1 using a driver layer to the drive module

\begin{tabular}{|c|c|c|}
\hline Number & Drive & Function Module \\
\hline 1 & Flash & S Flash Module \\
\hline 2 & E2PROM & E2PROM Module, To be completed \\
\hline 3 & FRAM & Ferroelectric memory module \\
\hline
\end{tabular}

iii.Functional layer: logical functions closely classify the instrument, at this level it is achieved by the application of layer to call. The functional layer is designed in accordance with the logic function will be closely linked logically functional design as a class, the functional layer is closely related to the specific application. In addition to some special design features can be fixed, but most of it need separately design alone and debugging; functional layer in the system is in the form of source code management and debugging; function organize the same functions in a source file; name the functional layer function to do a specific function prefix, easy to understand and recall. Table 2 functional modules used.

Figure 2 Software Hierarchy 
Table 2 functional layer used in function block

\begin{tabular}{|c|c|c|c|}
\hline Number & Features & Function Module & $\begin{array}{c}\text { Prefix } \\
\text { function }\end{array}$ \\
\hline 1 & $\begin{array}{c}\text { Protocol } \\
\text { processin } \\
\mathrm{g}\end{array}$ & $\begin{array}{c}\text { Statute for data analysis and } \\
\text { packaging }\end{array}$ & $\mathrm{Cmd}_{-}$ \\
\hline 2 & $\begin{array}{c}\text { azimuth } \\
\text { gamma }\end{array}$ & $\begin{array}{c}\text { azimuth gamma all algorithms } \\
\text { and processing }\end{array}$ & $\mathrm{AZG}_{-}$ \\
\hline 3 & $\begin{array}{c}\text { System } \\
\text { Configura } \\
\text { tion }\end{array}$ & $\begin{array}{c}\text { Associated with the system } \\
\text { configuration storage and } \\
\text { readout }\end{array}$ & SysCfg $_{-}$ \\
\hline 4 & $\begin{array}{c}\text { system } \\
\text { Conitorin } \\
\text { g and } \\
\text { recording }\end{array}$ & $\begin{array}{c}\text { Processing system, Operation, } \\
\text { Monitoring and recording system } \\
\text { health }\end{array}$ & ASys_ $_{-}$ \\
\hline
\end{tabular}

iv.Application Layer: The application management, in accordance with certain features designed to call the function layer, to achieve the management of the entire system. The main function is defined as a main (), to achieve the main process control, calling each function module, complete application functionality; logically classifying the operation to define and call functions using ASYS.

\section{Experimental Method}

I .Using gamma logging LWD azimuth to response for the simulation program experiments, numerical simulation for various of factors, improve various parameters of the logging methods, the factors into position, calliper clearance and ground conditions, by simulating the responding of the probe formation parameters, finding out the law, drawing response formula, better improve LWD azimuth detection parameter gamma logging method, lay the foundation for data processing. Using the principle of gamma photon interaction with the formation, the use of Monte Carlo simulation method, the LWD azimuth gamma logging tool window can be selected to achieve the desired effect can be the best to reflect the formation of information.

Ii .In both density quite different formations, respectively, from the detector and $137 \mathrm{Cs}$ radioactive source was simulated to obtain the optimum parameters of the length of the source pitch.

iii. Gamma logging LWD to establish azimuth the model using Monte Carlo methods, 137Cs radioactive source in a different density of the formation, the gamma field different litho strata distribution of numerical simulation, the $137 \mathrm{Cs}$ radioactive sources "back" shield, and between the radiation source and the detector, and thedistance between the probe are used shielding material is isolated, so $137 \mathrm{Cs}$ radioactive source emits gamma photons can be detected in the main instrument in accordance with the direction release, and it emits gammaphoton does not directly reach the detector from the borehole and received by the detector, and the impact detector count, and further information on the formation of judgment, to give this kind of distribution of radioactive sources, by setting the shield can achieve the best detection effect.
Iv .Borehole measurement conditions change, such as changing the hole diameter, changing the distance between the logging tool and the borehole wall, which gap (Standoff), etc., using Monte Carlo simulation method to study the condition of the wellbore LWD azimuth gamma Effect of horse logging methods; changing density, litho logy, thickness, interface parameters such as the formation, by the Monte Carlo simulation method to study gamma logging LWD azimuth respond to different formation conditions.

$\mathrm{V}$.The resulting application response relationship for dipping strata numerical simulation data by MATLAB, Origin8 and other computer languages, the resulting process simulation, the final azimuth of the LWD density to achieve imaging gamma logging; tilted to get under formation conditions Figure gamma imaging processing, determining formation dip and strata thickness, and use $\mathrm{C} \#, \mathrm{C}++$ formation processing software.

\section{Conclusion}

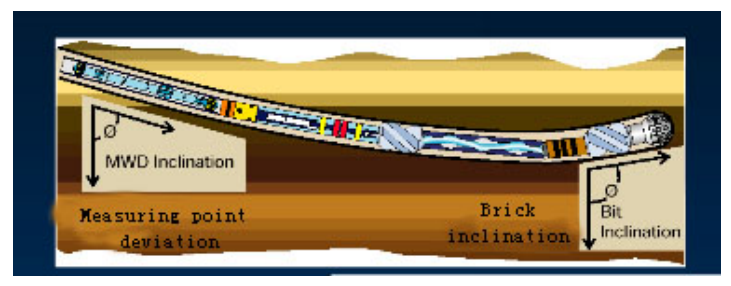

Figure 3 Embedded drilling azimuth gamma

Embedded gamma logging LWD azimuth LWD system in the process, as shown in Figure 3. Detecting the formation azimuth 16 , to obtain information on the data layer of $360^{\circ}$, using effective means of information and data imaging visual display of the detected formation. In the Monte Carlo model design, it will be divided into the wellbore surrounding the 16 sectors, the detector close to the wall, $22.5^{\circ}$ rotates once every stratum detect simulated to give gamma formations surrounding a borehole 16 in the direction of Ma photon counting rate, to give the corresponding information data formation processing. Then change to adifferent detection conditions calliper, litho logy, stratathickness, research azimuth gamma logging LWD a lot of log response .

Verification of embedded systems technology gamma LWD parameters are as follows

$>$ Measuring range: 0-500API

$>$ Gamma measurement accuracy: $\pm 2 \%$

$>$ Operating temperature: $0-150^{\circ} \mathrm{C}$

$>$ Sectorisation: 4-16 Sector

$>$ Real-time transmission: On gamma、Under gamma

$>$ Accuracy sector: $0.2^{\circ}$ (Static state), $2^{\circ}(180 \mathrm{RPM})$

$>$ Anti-vibration: $15 \mathrm{~g}$

$>$ Impact resistance: $500 \mathrm{~g}$

Embedded systems LWD Gamma has a very significant azimuth horizon interpretation advantages for thin reservoirs and complex formation, can greatly 
improve the drilling rate to enhance oil production. See Figure 4.

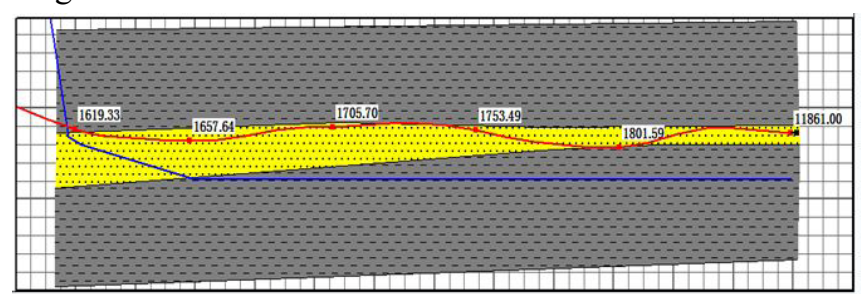

Figure 4 Embedded systems used in LWD oil sheet

Gamma

After several tests, this design parameters and function were verified. Reflects the embedded system by LWD gamma vibration is small, high precision, to ensure that the measured data in a composite drilling reliability.

\section{References}

[1] Liu Xinping, etc. LWD status quo and prospect data transmission technology [J]. Well Logging Technology, 2014, 32 (2) : 249-253.

[2] Paul Lurie, Philip Head, Jacke E S. Smart Drilling with Electric Drill String[c].SPE 79886, 2013.

[3]David Patrick Murphy .Advances in MWD and Formation valuation[J].World Oil, 2013, 226(3).

\section{About the Author:}

Zhou Yiren (1958-), male, Jiangxi Yichun, Nanchang Institute of Technology solid scientific research center, senior engineer, the main research directions: embedded systems. (Nanchang 330044)

Qiu Xiaolin, 1962.7, male, Jiangxi, Nanchang Institute of Technology Dean, Ph.D., professor, research direction: Materials Science (Nanchang 330044).

Guo Zhiqiang, 1991.9, male, Shanxi Datong, Nanchang Institute of Technology research laboratory, research direction: electromagnetic theory (Nanchang 330044) 